\title{
Probabilistic Chronological Production Simulation-Based Coordinated Dispatching for Cascaded Hydro-PV-PSH Combined Power Generation System
}

\author{
Shuai Zhang $\mathbb{D}^{D}$, Jingxian Yang $\mathbb{D}^{D}$, Hongjun Gao $\mathbb{D}^{D}$, Jichun Liu $\mathbb{D}$, and Junyong Liu $\mathbb{C}^{\circ}$ \\ College of Electrical Engineering, Sichuan University, Chengdu, China \\ Correspondence should be addressed to Jichun Liu; jichunliu@scu.edu.cn
}

Received 15 November 2020; Revised 2 December 2020; Accepted 11 December 2020; Published 30 December 2020

Academic Editor: Xiao-Shun Zhang

Copyright (c) 2020 Shuai Zhang et al. This is an open access article distributed under the Creative Commons Attribution License, which permits unrestricted use, distribution, and reproduction in any medium, provided the original work is properly cited.

\begin{abstract}
The comprehensive and effective utilization of multiple renewable sources involving water, solar, and other energies have been receiving more and more attention, and the coordinated dispatching considering multiple uncertainties has become one of the research focuses currently. To cope with the stochastic dispatching of cascaded hydro-PV-PSH (pumped storage hydropower, PSH) complementary power generation system, this paper has proposed a two-stage optimized dispatching method combining uncertainties simulation and complementary dispatching schemes optimization. Firstly, a modified PCPS (probabilistic chronological production simulation) method is proposed to simulate the uncertain power output of each energy type, and thus the complementary dispatching schemes and performance parameters are obtained. In the simulation process, a special treatment method combining overall uncertainties, overall dispatching measures, and interstage distribution for cascaded hydropower stations is given out. Secondly, a dual-objective optimized model considering reliability and economic performance and MOQPSO algorithm is introduced to calculate the optimal Pareto solution set of stochastic dispatching schemes. The results of the case study verified the feasibility and calculating performance of the proposed method in developing optimized dispatching schemes.
\end{abstract}

\section{Introduction}

Facing the global energy crisis, the comprehensive utilization of renewable energies involving solar, wind, hydropower, biomass, geothermal energy, and so forth [1] might be a feasible solution. The research of hybrid power generation of multiple renewable energies has achieved lots of remarkable progress reflected in the following aspects. The designing and planning of HREGS (hybrid renewable energy generation system, HREGS) is taken as a research focus, and it contains optimal overall sizing [2] and unit sizing [3] of the hybrid system, configurations [4-6] and placement [7] of each unit, and optimal planning and design [4]. Various analyses on HREGS such as cost $[3,8]$ and technoeconomic $[9,10]$ analysis, performance $[11]$ and feasibility analysis, market evaluation, environmental and social benefits analysis, reliability $[10,12]$ and power quality assessment, and so forth are another research interest. The business model [13] of HREGS is an emerging research hotspot recently. The stochastic and intermittent nature of renewables and the complex coupling of multiple uncertainties from the hybrid power system have become the largest barriers to the utilization of renewables [14, 15]. Therefore, aspects of modeling of the hybrid system $[4,15]$, uncertain output forecasting [16], multirenewable energies management [17], control [5, 6], dispatching $[18,19]$, and operation [20] strategies, which are different from general topics, should be taken seriously to research with more investment. PSH (pumped storage hydropower, PSH), one of the high-performance power generation sources, has excellent capabilities of rapid response and flexible regulation and is widely used in renewable power systems [21]. Its usage scenario of mitigating output variation of the hybrid power system and effect evaluation is displayed in [22]. Literature [5, 23] has also 
presented the challenges, barriers, and future vision of the development and application of HREGS involving PSH. In conclusion, facing the multiple uncertainties and the complex coupling of HREGS, PSH might be a proper solution to the uncertainties. Therefore, how to realize the optimal operation and formulate dispatching strategies for HREGS is of great value to research.

Due to different random and intermittent natures of different renewables, the hybrid power generation system cannot be dispatched with the conventional manner applied on thermal power and diesel generators [24]. Moreover, operational problems such as frequency excursions and system stability cannot be addressed effectively only by conventional AGC $[19,25,26]$. Therefore, an optimal dispatching strategy is needed and implemented to address the problems. The general dispatching optimization techniques are divided into two major types including mathematical procedures and heuristic optimization [18] which are concerned with finding an optimal dispatching strategy considering the operation or system constraints. Almost each general technique only focuses on a specific objective task and has performed a bad generalization capability [27]. In current studies, the multiple uncertainties of renewables and their coupling cannot be taken into account and handled properly. Techniques of day-ahead and real-time optimal power flow are introduced to obtain the optimal dispatching strategy for power systems with considerable RERs (renewable energy resources) [28]. But the variabilities and uncertainties introduced by renewable energy are usually simply substituted with frozen static snapshot forecast values or nominal forecast values which leads to a result that the optimization model has turned into a deterministic model. Reference [29] has proposed a chance-constrained optimal power flow technique to account for the uncertainty of renewables to obtain the optimal load ensemble control strategy, but its application in the dispatching of HREGS still needs more exploration. Adaptive robust optimization for the economic dispatch of power systems with renewables highly penetrated is presented in [30], and the temporal and spatial correlation of uncertainty is modeled by using the idea of dynamic uncertainty sets. In [31], an idea of integrating the risk of renewable's uncertainty into profitability assessments for investors and regarding renewables as risky assets to be invested based on portfolio theory is proposed to obtain an optimal solution to the configuration and dispatching issues of HREGS. The idea is valuable, but the model is simply depicted, and extensive investigation is needed to be conducted on it. A method based on regression techniques and Chebyshev's inequality is introduced in [32] to calculate the dispatching or displacement ratio of the conventional generators under various contingencies from renewables. But its application in HEGES still needs much valuable research.

PPS (probabilistic production simulation) [33] is a powerful technique for power generation system, and the uncertainties are considered as random failure probability. Relevant data including power generation expectation, unit power generation cost, and generation reliability can be obtained by this technique. It is widely used in power system cost analysis, planning and configuration, price setting, and reliability evaluation [34]. The basis of PPS is to transform the time-sequence load curve into ELDC (equivalent load duration curve), and the time-sequence related information and constraints are ignored. This will bring barriers to the dynamic operation constraints and the calculating accuracy cannot be guaranteed. Besides, a heavy computational burden will emerge due to a large number of convolution and deconvolution calculations [35]. Given the drawbacks of the PPS technique when applied to HREGS, a modified PPS technique considering the chronological characteristics of the system [36], named PCPS (probabilistic chronological production simulation) technique, is introduced to optimize the coordinated dispatch strategy for HREGS, and the uncertainties of multiple renewables are properly formulated by random failure probability. The PCPS technique could handle dynamic chronological constraints directly and obtain accurate reliability data efficiently. This technique provides an outstanding solution to the multiple uncertainties and complex coupling of HREGS.

In this paper, the joint complementary power generation system consisting of cascaded hydropower, PV, and PSH is taken as the research object to study the coordinated dispatching strategy of the complementary system composed of multiple renewables. Due to the different natures of each renewable type, different PCPS methods are proposed for cascaded hydropower, PV, and PSH respectively. Then, the comprehensive framework of PCPS for the hybrid system is obtained, and the dispatching strategy set is obtained through the coordinated PCPS technique. On this basis, a dual-objective optimal dispatching model considering reliability and economic performance is proposed, and the MOQPSO algorithm is introduced to calculate the optimal Pareto dispatching solution set.

The paper is organized as follows. Section 2 presents the coordinated PCPS method for the cascaded hydro-PV-PHS combined power generation system. Section 3 displays the dual-objective dispatching model and the solution method based on the MOQPSO algorithm. Section 4 introduces a case study to verify the effectiveness and calculating efficiency of the proposed method. Finally, Section 5 concludes the paper.

\section{Coordinated PCPS Methods for the Complementary Power Generation System}

Owing to the different natures of PV, cascaded hydropower, and PSH, the PCPS method suitable for each generation type has many differences. In this part, the PCPS method for each renewable will be presented respectively. On this basis, a comprehensive PCPS flow path of the complementary power generation system is given out.

2.1. PCPS for PV. PV output is divided into two parts: deterministic output and random output cuts, and the actual output is the difference between the deterministic output and random cuts. In the deterministic output, solar radiation 
is the most influential factor, and a direct ratio exists between deterministic output and solar radiation intensity as expressed in formula (1). Other factors such as weather variation and temperature bring about random output cuts due to their strong uncertainties that reduce PV output by weakening deterministic output. Models of deterministic output and random output cuts are presented, respectively, as follows:

$$
P_{c, t}=P_{\mathrm{st}} \cdot \frac{I_{t}}{I_{\mathrm{st}}},
$$

where $P_{c, t}$ is the deterministic output; $P_{s t}$ is power output in standard conditions (solar radiation intensity $I_{\text {st }}$ is $1000 \mathrm{~W} /$ $\mathrm{m}^{2}$, and the temperature is $25^{\circ} \mathrm{C}$ ); $I_{t}$ is the maximum value of solar radiation intensity reaching the ground without occlusion; $I_{\text {st }}$ is the solar radiation intensity in standard conditions.

2.1.1. Modeling for Deterministic Output. The radiation intensity from the sun directly radiated to the atmosphere $I_{0}$ is only related to the relative position between the sun and the Earth [37], and the value can be calculated from formula (2). The solar radiation reaching the ground can be divided into direct radiation and diffuse radiation, and the transparency coefficient is introduced to describe the weakening effect of the atmosphere on solar radiation. The transparency of direct radiation can be obtained from formulas (3)-(6) [38], and the direct radiation intensity is shown in formula (7):

$$
\begin{aligned}
I_{0} & =S_{0}\left(1+0.033 \cos \left(\frac{2 \pi(N+10)}{365}\right)\right) \\
\tau_{\text {dir }} & =0.56\left(e^{-0.56 M_{h}}+e^{-0.095 M_{h}}\right) \\
M_{h} & =\left(\sqrt{1229+(614 \sin \alpha)^{2}}-614 \sin \alpha\right) \cdot\left(\frac{288-0.0065 h}{288}\right)^{5.256}
\end{aligned}
$$

$\sin \alpha=\sin \delta \sin \phi+\cos \delta \cos \phi \cos \omega$,

$$
\begin{aligned}
\delta & =\frac{2 \pi \cdot 23.45^{\circ}}{360^{\circ}} \cdot \sin \left(\frac{2 \pi(284+N)}{365}\right), \\
I_{\text {dir }} & =I_{0} \tau_{\text {dir }} \sin \alpha,
\end{aligned}
$$

where $I_{0}$ is the radiation intensity directly radiated to the atmosphere from the sun; $S_{0}$ is the solar constant which represents the total amount of solar radiation entering the Earth's atmosphere per unit area; $N$ represents the daily sequence; $\tau_{\text {dir }}$ is the transparency coefficient of direct radiation; $M_{h}$ is air mess, a function of altitude and altitude angle of the sun; $h$ is the altitude of the specified location; $\alpha$ is the altitude angle of the sun; $\phi$ is the latitude of the specified location; $\omega$ is the hour angle of the sun, related to the time of the specified day; and $I_{\text {dir }}$ is the direct radiation intensity of the sun.

The diffuse radiation is related to multiple weather conditions, and existing experiments show that the transparency coefficient of direct radiation is approximated as a linear relationship with that of diffuse radiation [39] shown in formula (8), and the diffuse radiation intensity can be calculated by formula (9):

$$
\begin{aligned}
\tau_{\mathrm{dif}} & =0.271-0.274 \tau_{\mathrm{dir}}, \\
I_{\mathrm{dif}} & =\frac{\sin \alpha}{2} \cdot \frac{1-\tau_{\mathrm{dif}}}{1-1.4 \ln \left(\tau_{\mathrm{dif}} / M_{h}\right)} k,
\end{aligned}
$$

where $\tau_{\text {dif }}$ is the transparency coefficient of diffuse radiation; $I_{\text {dif }}$ is diffuse radiation intensity of the sun; and $k$ is a parameter related to $M_{h}$, and its value range is $[0.60,0.90]$.

In summary, ignoring other random factors, the total solar radiation intensity $I_{t}$ at a certain position and at a certain time is obtained by formula (10), and the deterministic output of PV $P_{c, t}$ can be achieved if $I_{t}$ is brought into formula (1):

$$
I_{t}=I_{\mathrm{dir}}+I_{\mathrm{dif}}
$$

2.1.2. Modeling for Random Output Cuts. The reduction factor $\eta_{t}$, the relative difference between the actual output and the deterministic output, is introduced to quantify the weakening effect of the randomness of shadows, cloud cover, weather variation, temperature, and so forth . According to the statistical research [40], it is believed that the probability density function of $\eta_{t}$ conforms to beta distribution:

$$
\eta_{t} \sim B e(\alpha, \beta)
$$

Based on the probability density function of $\eta_{t}$, the uncertain PV output cuts can be expressed into several states; meanwhile, $\eta_{t}$ and its probability $p_{t}$ of each state is obtained. The PV output $P_{t}^{\mathrm{PV}}$ and its expectation $\mathrm{EP}_{t}^{\mathrm{PV}}$ are given by the following formulas (12) and (13):

$$
\begin{aligned}
P_{t}^{\mathrm{PV}} & =\left(1-\eta_{t}\right) P_{c, t}, \\
\mathrm{EP}_{t}^{\mathrm{PV}} & =\left(1-\sum_{n=1}^{N} \eta_{t, n} P_{t, n}\right) P_{c, t} .
\end{aligned}
$$

2.1.3. Simulation Data of $P V$. In the hybrid system, the total power load is $L_{t}^{0}$, and the power load of the PV station is set as $L_{t}^{\mathrm{PV}}$. After receiving power supply service from $\mathrm{PV}$, the remaining load in time sequence is described as formula (14), and the corresponding reliability indexes are obtained by formulas (15) and (16): 


$$
\begin{aligned}
& L_{t}^{\prime}=\left\{\begin{array}{l}
L_{t}^{0}-\left(1-\sum_{n=1}^{N} \eta_{t, n} p_{t, n}\right) P_{c, t}, \quad L_{t}^{\mathrm{PV}} \geq P_{c, t} \\
L_{t}^{0}-\sum_{n=1}^{N_{1}}\left(1-\eta_{t, n}\right) p_{t, n} P_{c, t}-\sum_{n=N_{1}+1}^{N} p_{t, n} L_{t}^{\mathrm{PV}}, \quad L_{t}^{\mathrm{PV}}<P_{c, t}
\end{array}\right. \\
& \operatorname{LOLP}_{t}^{\mathrm{PV}}=\frac{\Delta t}{T} \sum_{n=1}^{N_{1}} p_{t, n}, \\
& \mathrm{EENS}_{t}^{\mathrm{PV}}=\Delta t \cdot\left[L_{t}^{\mathrm{PV}}-\left(1-\sum_{n=1}^{N_{1}} \eta_{t, n} P_{t, n}\right) P_{c, t}\right]
\end{aligned}
$$

where $L_{t}^{\prime}$ is the remaining power load after receiving PV power; $N$ is the number of PV output state; $N_{1}$ is the number of the states that $\mathrm{PV}$ output is lower than $L_{t}^{\mathrm{PV}}$, and if $L_{t}^{\mathrm{PV}} \geq P_{c, t}, N_{1}$ is equal to $N$; $\operatorname{LOLP}_{t}^{\mathrm{PV}}$ is the loss of load probability; $\mathrm{EENS}_{t}^{\mathrm{PV}}$ is the expectations of energy not served.

2.2. PCPS for Cascaded Hydropower. As the probabilistic output of cascaded hydropower at each stage is affected by many deterministic and uncertain factors such as installed capacity, net flow, reservoir capacity, water delay, interstage constraints, failure outage, evaporation, and leakage, direct modeling for cascaded hydropower is of great difficulty. Therefore, an indirect way is proposed to determine the equivalent available capacity of the entire cascaded system, and then a power capacity interstage distribution plan is developed. In this way, the PCPS for cascaded hydropower is accomplished.

\subsubsection{Equivalent Available Capacity of the Entire Cascaded} System. Take the greatest common divisor $C_{i}$ of the installed capacities of the stations in the cascaded system as the capacity of the generation unit, and its randomness is described as a two-stage model expressed in formula (17). The moment $M_{i}$ and cumulant $\mathrm{GC}_{i, r}$ are given by formulas (18) and (19), respectively. According to the properties of cumulant, the cumulant of equivalent available capacity EGC of the first $k$ units can be expressed as the sum of cumulant of each unit shown in formula (20), and the probability distribution function $F\left(z_{1}\right)$ and the probability density function of the first $k$ units' equivalent available capacity based on Edgeworth series are obtained by formulas (21)-(27):

$$
\begin{aligned}
A_{i} & = \begin{cases}C_{i}, & p_{t, i}=1-q_{i}, \\
0, & p_{t, i}=q_{i},\end{cases} \\
M_{i, r} & =\left(1-q_{i}\right) \cdot C_{i}^{r},
\end{aligned}
$$

$$
\begin{aligned}
\left\{\begin{array}{l}
\mathrm{GC}_{i, 1}=M_{i, 1}, \\
\mathrm{GC}_{i, r+1}=M_{i, r+1}-\sum_{j=1}^{r} \frac{r !}{j !(r-j) !} M_{i, r} \cdot \mathrm{GC}_{i, r-j+1}
\end{array}\right. \\
\mathrm{EGC}_{k, r}=\sum_{i=1}^{k} \mathrm{GC}_{i, r}, \\
F(z)=\int_{-\infty}^{z} N\left(z_{1}\right) \mathrm{d} z_{1}+\frac{\mathrm{EGC}_{k, 3}}{3 !\left(\mathrm{EGC}_{k, 2}\right)^{3 / 2}} \cdot \frac{\mathrm{d}^{2} N(z)}{\mathrm{d} z} \\
\quad-\frac{\mathrm{EGC}_{k, 4}}{4 !\left(\mathrm{EGC}_{k, 2}\right)^{2}} \cdot \frac{\mathrm{d}^{3} N(z)}{\mathrm{d} z}-\frac{10\left(\mathrm{EGC}_{k, 3}\right)^{2}}{6 !\left(\mathrm{EGC}_{k, 2}\right)^{3}} \cdot \frac{\mathrm{d}^{5} N(z)}{\mathrm{d} z} \\
z=\frac{\left(x-\mu_{k}\right)}{\sigma_{k}}=\frac{\left(x-\mathrm{EGC}_{k, 1}\right)}{\sqrt{\mathrm{EGC}_{k, 2}}} \\
N(z)= \\
\frac{e^{-\left(z^{2} / 2\right)}}{\sqrt{2 \pi}},
\end{aligned}
$$

$\frac{\mathrm{d}^{2} N(z)}{\mathrm{d} z}=\left(z^{2}-1\right) N(z)$,

$\frac{\mathrm{d}^{3} N(z)}{\mathrm{d} z}=\left(-z^{3}+3 z\right) N(z)$,

$\frac{\mathrm{d}^{5} N(z)}{\mathrm{d} z}=\left(-z^{5}+10 z^{3}-15 z\right) N(z)$,

$$
f(z)=\frac{\mathrm{d} F(z)}{\mathrm{d} z}
$$

where $A_{i}$ and $q_{i}$ are the available capacity and the outage rate of the $i^{\text {th }}$ power unit; $z$ is the standardized variable of the equivalent available capacity of the first $k$ units; and $N(z)$ is the probability density function of normal distribution.

2.2.2. Simulation Data of the Cascaded System. According to the probability density function $f(z)$, the equivalent available capacity $P_{t}$ and its probability $p_{t}$ can be obtained. The power load of the whole cascaded system is set as $L_{t}^{\mathrm{CH}}$, and the expected power value of the cascaded system $\mathrm{EP}_{t}$ is given by formula (28). From the relationship between the probability distribution of random variable $x$ and its probability density, formulas (29)-(31) can be obtained, and take them into formula (28), then formula (28) will be transformed in the form of formula (32): 


$$
\begin{gathered}
\mathrm{EP}_{t}^{\mathrm{CH}}=\int_{0}^{L_{t}^{\mathrm{CH}}} x \cdot f(x) \mathrm{d} x+L_{t}^{\mathrm{CH}} \cdot \int_{L_{t}^{\mathrm{CH}}}^{\infty} f(x) \mathrm{d} x, \\
F(x)=\int_{-\infty}^{x} f(x) \mathrm{d} x=\int_{0}^{x} f(x) \mathrm{d} x, \\
\int_{0}^{L_{t}^{\mathrm{CH}}} x \cdot f(x) \mathrm{d} x=L_{t}^{\mathrm{CH}} F\left(L_{t}^{\mathrm{CH}}\right)-\int_{0}^{L_{t}^{\mathrm{CH}}} F(x) \mathrm{d} x, \\
\int_{L_{t}^{\mathrm{CH}}}^{\infty} f(x) \mathrm{d} x=1-F\left(L_{t}^{\mathrm{CH}}\right), \\
\mathrm{EP}_{t}^{\mathrm{CH}}=L_{t}^{\mathrm{CH}}-\int_{0}^{L_{t}^{\mathrm{CH}}} F(x) \mathrm{d} x .
\end{gathered}
$$

The remaining power load $L_{t}^{\prime}$ after receiving the cascaded system power is given by formula (33), and the reliability indexes $\mathrm{LOLP}_{t}, \mathrm{EENS}_{t}$ are obtained by formulas (34) and (35):

$$
\begin{aligned}
L_{t}^{\prime} & =L_{t}^{0}-\int_{0}^{L_{t}^{\mathrm{CH}}} x \cdot f(x) \mathrm{d} x-L_{t}^{\mathrm{CH}} \cdot \int_{L_{t}^{\mathrm{CH}}}^{\infty} f(x) \mathrm{d} x \\
& =L_{t}^{0}-L_{t}^{\mathrm{CH}}+\int_{0}^{L_{t}^{\mathrm{CH}}} F(x) \mathrm{d} x, \\
\operatorname{LOLP}_{t}^{\mathrm{CH}} & =\frac{\Delta t}{T} \int_{0}^{L_{t}^{\mathrm{CH}}} f(x) \mathrm{d} x=\frac{\Delta t}{T} F\left(L_{t}^{\mathrm{CH}}\right), \\
\mathrm{EENS}_{t}^{\mathrm{CH}} & =\Delta t\left(L_{t}^{\mathrm{CH}}-\mathrm{EP}_{t}\right)=\Delta t \cdot \int_{0}^{L_{t}^{\mathrm{CH}}} F(x) \mathrm{d} x .
\end{aligned}
$$

\subsubsection{Power Capacity Interstage Distribution Method.} The target of power capacity distribution is to maximize energy storage and minimize the loss of energy. Set the total natural runoff power output of the cascaded system as $P_{t}^{\text {runoff }}$; if $P_{t}^{\text {runoff }}>L_{t}^{\mathrm{CH}}$, the cascaded system needs to store the extra energy, or the system needs to release more water to compensate for power output.

When $P_{t}^{\text {runoff }}<L_{t}^{\mathrm{CH}}$, it needs the $i-$ th reservoir to release water to compensate for the power output shortage, and the head loss $\Delta H_{i}^{\text {loss }}$ in this course is given by formula (36). Take $K=A_{i} \cdot \sum_{j=i}^{M} H_{j}$ as a discriminant coefficient, and it is clear that a large value of $K$ indicates that the head loss caused by per unit power output is smaller. Therefore, the reservoir with a large discriminant coefficient should be given priority to release water for the power output supplement:

$$
\begin{aligned}
\Delta H_{i}^{\text {loss }} & =\frac{\left(L_{t}^{\mathrm{CH}}-P_{t}^{\text {runoff }}\right) \cdot \Delta t}{\eta \cdot A_{i} \cdot \sum_{j=i}^{M} H_{j}}, \\
\Delta H_{i}^{\text {increase }} & =\frac{\left(P_{t}^{\text {runoff }}-L_{t}^{\mathrm{CH}}\right) \cdot \Delta t}{A_{i} \cdot \sum_{j=i}^{M} H_{j}},
\end{aligned}
$$

where $A_{i}$ is the cross-sectional area of the $i^{\text {th }}$ reservoir; $\sum_{j=i}^{M} H_{j}$ is the total head of the $i^{\text {th }}$ reservoir and its downstream reservoirs.

Similarly, when $P_{t}^{\text {runoff }} \geq L_{t}^{\mathrm{CH}}$, the extra energy should be stored in the $i-$ th reservoir, and the head increase $\Delta H_{i}^{\text {increase }}$ is given by formula (37). $A_{i} \cdot \sum_{j=i}^{M} H_{j}$ in formula (37) is also regarded as a discriminant coefficient, and a small value of it will realize a large $\Delta H_{i}^{\text {increase }}$ caused by per unit energy. Therefore, the reservoir with a small discriminant coefficient should be given priority to store extra energy.

The main steps of the interstage power capacity distribution based on the discriminant coefficient are listed as follows.

(1) Calculate the total natural runoff power output $P_{t}^{\text {runoff }}$, and the discriminant coefficients of each reservoir in the cascaded system.

(2) If $P_{t}^{\text {runoff }}<L_{t}^{\mathrm{CH}}$, the hydropower station with the largest discriminant coefficient is ordered to generate electricity first, and the other stations generate electricity with its coming flow. If the load power demand cannot be met, the rest stations will release water in turn in order of the discriminant coefficient from large to small for power generation until the load demand balance is realized or the reservoirs are all in the lowest water storage level.

(3) If $P_{t}^{\text {runoff }} \geq L_{t}^{\mathrm{CH}}$, the hydropower station with the smallest discriminant coefficient is ordered to generate electricity with part of the coming flow, and the rest flow will be stored in the reservoir. The other stations generate electricity with its coming flow. If the water level of the working reservoir reaches the maximum and the extra energy still exists, reservoirs of the rest stations will be arranged in turn to store water in the order of discriminant coefficient from small to large until all the extra energy is stored or all the reservoirs reached the highest water storage level.

Due to the lack of regulating reservoirs of runoff hydropower stations, they do not possess water supplement and storage ability. This kind of power station is believed that its equivalent cross-sectional area is infinite, and the value of $K$ is infinite. According to the distribution strategy aforementioned, when it needs to store energy, the runoff stations implement no action throughout the whole course; when it needs to compensate for power generation, the runoff stations will be arranged to generate electricity in priority.

2.3. PCPS for PSH. PSH is not affected by runoff flow, water delay, and other uncertain factors. It can realize the functions of peak regulation, frequency adjustment, emergency standby, and so forth under dispatching orders. The random forced outage rate of each unit of PSH is taken as the main uncertain factor here, and the probabilistic discrete available capacity of PSH is displayed by formula (38). Supposing that the $n$ units of PSH have the same installed capacity $c$ and the same forced outage rate $q$, the probabilistic available capacity $c_{i}$ of PSH and its probability $a_{i}$ is obtained from formulas (39) and (40): 


$$
\begin{gathered}
\left\{c_{1} a_{1}, c_{2} a_{2}, \ldots, c_{m} a_{m}\right\}, \\
c_{i}=i c, \quad i=0 \sim n, \\
a_{i}=(1-q)^{i} q^{n-i} \frac{n !}{i !(n-i) !} .
\end{gathered}
$$

The simulation of the power generation process of $\mathrm{PSH}$ is the same as that of the conventional power generation unit. The load undertaken by PSH is set as $L_{t}^{\mathrm{PSG}}$, and the expected power output $\mathrm{EP}_{t}^{\mathrm{PSG}}$ at this moment is obtained by formula (41). The remaining load $L_{t}^{\prime}$ is calculated by formula (42), and the reliability indexes $\operatorname{LOLP}_{t}^{\mathrm{PSG}}, \mathrm{EENS}_{t}^{\mathrm{PSG}}$ are obtained by formulas (43) and (44):

$$
\begin{aligned}
\mathrm{EP}_{t}^{\mathrm{PSG}} & =\sum_{i=1}^{m} a_{i} c_{i}+L_{t}^{\mathrm{PS}} \sum_{i=m+1}^{n+1} a_{i}, \\
L_{t}^{\prime} & =L_{t}^{0}-\sum_{i=1}^{m} a_{i} c_{i}-L_{t}^{\mathrm{PS}} \sum_{i=m+1}^{n+1} a_{i}, \\
\mathrm{LOLP}_{t}^{\mathrm{PSG}} & =\frac{\Delta t}{T} \sum_{i=1}^{m} a_{i}, \\
\mathrm{EENS}_{t}^{\mathrm{PSG}} & =\Delta t\left(L_{t}^{\mathrm{PS}}-\sum_{i=1}^{m} a_{i} c_{i}-L_{t}^{\mathrm{PS}} \sum_{i=m+1}^{n+1} a_{i}\right),
\end{aligned}
$$

where $m$ is the number of the available generation capacity states and is determined by $c_{m}<L_{t}^{\mathrm{PS}}<c_{m+1}$.

The pumping process of PSH is used for excess energy storage, and it occurs when the expected output of PV, the total natural runoff power of the cascaded system, or the sum of the two is greater than the total load demand. If the excess power used for pumping is set as $P_{t}^{\text {pump }}$, the expected pumping capacity $\mathrm{EP}_{t}^{\mathrm{PSP}}$ of $\mathrm{PSH}$ is obtained by the following formula:

$$
\mathrm{EP}_{t}^{\mathrm{PSP}}=\sum_{i=1}^{k} a_{i} c_{i}+P_{t}^{\mathrm{pump}} \sum_{i=k+1}^{n+1} a_{i}
$$

where $k$ is the number of the available pumping capacity states and is determined by $c_{k}<P_{t}^{\text {pump }}<c_{k+1}$.

2.4. The Comprehensive PCPS Flow. Without concerning power generation cost, the specified load demand can be burdened by anyone or any combination of PV, cascaded system, and PSH. If the load demand could not be met, another power source is arranged to generate until the load demand is minimized. If the load demand in a certain period is lower than each of or the sum of the expected PV output and the total natural runoff power of the cascaded system, the excess power should be stored in PSH system by pumping water. Reliability indexes such as LOLP, EENS of each generation unit can be obtained by the proposed method, and the total generation reliability indexes of this period are given by the following formulas:

$$
\mathrm{LOLP}_{t}=1-\left(1-\mathrm{LOLP}_{t}^{\mathrm{PV}}\right) \cdot\left(1-\mathrm{LOLP}_{t}^{\mathrm{CH}}\right) \cdot\left(1-\mathrm{LOLP}_{t}^{\mathrm{PSG}}\right)
$$

$$
\mathrm{EENS}_{t}=\Delta t \cdot\left[\begin{array}{l}
L_{t}-\left(1-\sum_{n=1}^{N_{1}} \eta_{t, n} p_{t, n}\right) P_{c, t} \\
-\int_{0}^{L_{t}^{\mathrm{CH}}} F(x) \mathrm{d} x-\left(\sum_{i=1}^{m} a_{i} c_{i}+L_{t}^{\mathrm{PS}} \sum_{i=m+1}^{n+1} a_{i}\right)
\end{array}\right]
$$

where $\operatorname{LOLP}_{t}^{\mathrm{PV}}, \mathrm{LOLP}_{t}^{\mathrm{CH}}$, and $\operatorname{LOLP}_{t}^{\mathrm{PSG}}$ are the loss of load probabilities of PV, cascaded hydropower system, and $\mathrm{PSH}$, respectively.

The comprehensive probabilistic chronological production simulation flow is displayed in Figure 1.

\section{Dual-Objective Optimized Dispatching Model}

Based on the PCPS method, the generation dispatching scheme set that meets coordinated complementarity is obtained. Meanwhile, the reliability and economic indexes of each scheme are also obtained. In this section, a dual-objective dispatching model is proposed to seek the equilibrium scheme to balance the reliability and economic performance of the scheme. MOQPSO (Multiobjective Quantum-behaved Particle Swarm Optimization) algorithm is introduced to calculate the optimal Pareto solution set of the dispatching model.

In the dispatching model, the minimum probability of load loss and the minimum operating cost are taken as the dual objectives as follows:

$$
\begin{aligned}
& \min \operatorname{LOLP}_{i, t}, \\
& \min C_{i, t}^{\mathrm{ope}}=C_{i, t}^{G}+C_{i, t}^{\mathrm{EN}}+C_{i, t}^{\mathrm{cur}}, \\
& C_{i, t}^{G}=C_{i, t}^{\mathrm{CH}}+C_{i, t}^{\mathrm{PV}}+C_{i, t}^{\mathrm{PS}}
\end{aligned}
$$$$
C_{i, t}^{G}=C_{i, t}^{\mathrm{CH}}+C_{i, t}^{\mathrm{PV}}+C_{i, t}^{\mathrm{PSG}}+C_{i, t}^{\mathrm{PSP}},
$$

where $\operatorname{LOLP}_{i, t}$ and $C_{i, t}^{\text {ope }}$ are the loss of load probability and the operating cost of the $i^{\text {th }}$ scheme. $C_{i, t}^{\text {ope }}$ is composed of three parts: power generation cost $C_{i, t}^{G}$, penalty cost for energy not served $C_{i, t}^{\mathrm{EN}}$, and penalty cost for energy curtailment $C_{i, t}^{\mathrm{cur}} \cdot C_{i, t}^{\mathrm{CH}}$, $C_{i, t}^{\mathrm{PV}}, C_{i, t}^{\mathrm{PSG}}$, and $C_{i, t}^{\mathrm{PSP}}$ are operating cost for cascaded hydropower, $\mathrm{PV}$ unit, power generation of $\mathrm{PSH}$, and water pumping of $\mathrm{PSH}$, respectively. $c_{i, t}^{\mathrm{CH}}, c_{i, t}^{\mathrm{PV}}, c_{i, t}^{\mathrm{PSG}}$, and $c_{i, t}^{\mathrm{PSP}}$ are price 


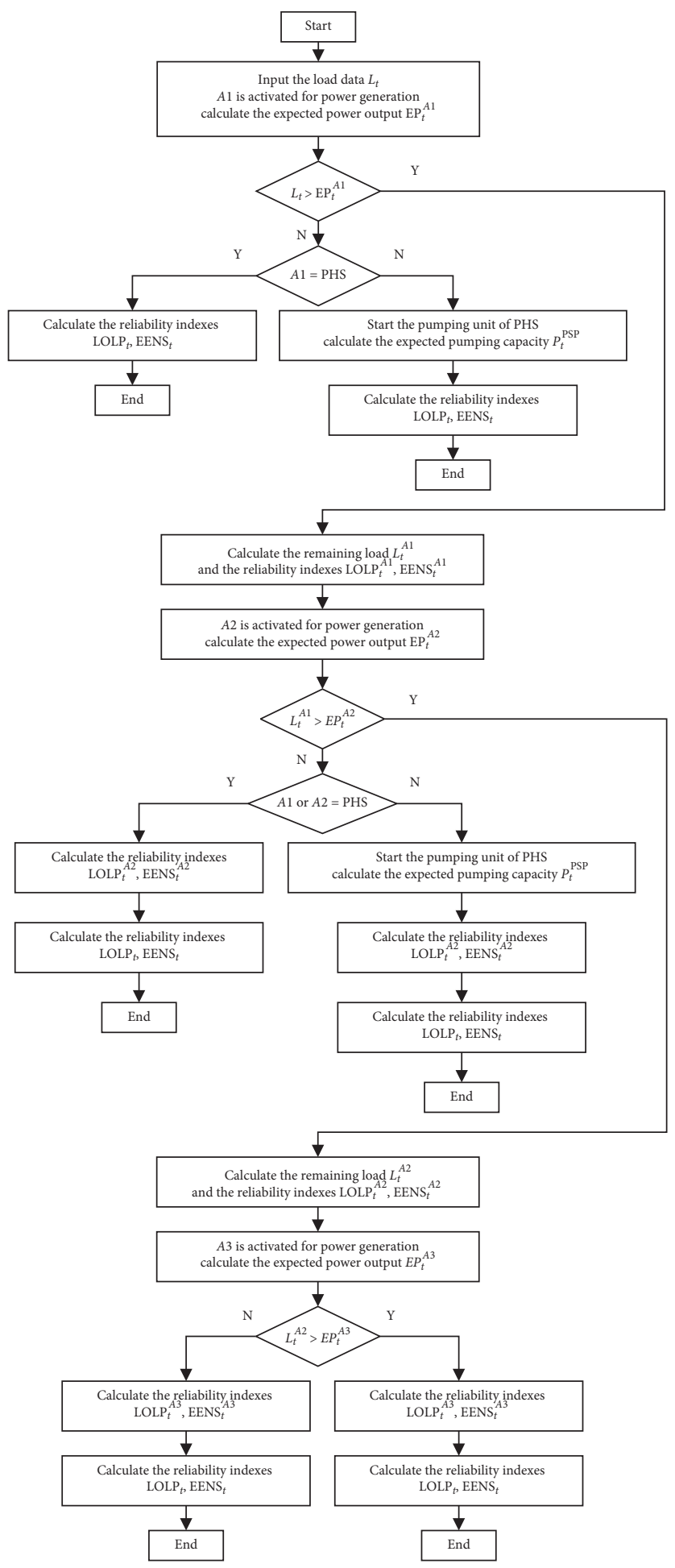

Figure 1: Probabilistic chronological production simulation flow.

coefficients of cascaded hydropower, PV unit, power generation of pumped storage, and water pumping of pumped storage, respectively. $z_{i, t}^{\mathrm{PSG}}$ and $z_{i, t}^{\mathrm{PSP}}$ are operation status variables of PSH. When $z_{i, t}^{\mathrm{PSG}}$ equals 1 or $z_{i, t}^{\mathrm{PSP}}$ equals 0 , the system is in generation status or else in pumping status.

The operation constraints of the hybrid system are mainly listed as follows:
3.1. Cascaded Hydropower Operation Constraints. Operation constraints of cascaded hydropower mainly include reservoir capacity constraint (53), hydropower output constraint (54), discharged water flow constraint (55), water balance constraint (56), interstage hydraulic connection constraint (57), and ramp rate constraint (58): 


$$
\begin{aligned}
V_{k}^{\mathrm{min}} & \leq V_{k, t} \leq V_{k}^{\mathrm{max}}, \\
P_{k, t}^{\mathrm{CH} \text { min }} & \leq P_{k, t}^{\mathrm{CH}} \leq P_{k, t}^{\mathrm{CH} \max }, \\
Q_{k}^{\mathrm{CH} \text { min }} & \leq Q_{k, t}^{\mathrm{CH}} \leq Q_{k}^{\mathrm{CH} \max }, \\
V_{k, t+1} & =V_{k, t}+\left(Q_{k, t}^{\text {in }}-Q_{k, t}^{\text {out }}\right) \Delta t \\
& =V_{k, t}+\left(Q_{k, t}^{\text {in }}-Q_{k, t}^{\mathrm{Gen}}-Q_{k, t}^{\mathrm{Cur}}\right) \Delta t, \\
Q_{k+1, t+\tau}^{\text {in }} & =Q_{k, t}^{\text {out }}+Q_{k, t}^{\text {int }}, \\
\delta^{L} \Delta t & \leq P_{k, t+1}^{\mathrm{CH}}-P_{k, t}^{\mathrm{CH}} \leq \delta^{U} \Delta t,
\end{aligned}
$$

where $V_{k, t}, V_{k}^{\min }, V_{k}^{\max }$ are the water storage of the $k^{\text {th }}$ reservoir and its minimum and maximum value; $P_{k, t}^{\mathrm{CH}}$, $P_{k, t}^{\mathrm{CH} \min }, P_{k, t}^{\mathrm{CH} \max }$ are the power output and its minimum and maximum limitations of the $k^{\text {th }}$ hydropower station in the cascaded system; $Q_{k, t}^{\mathrm{CH}}, Q_{k}^{\mathrm{CH} \text { min }}, Q_{k}^{\mathrm{CH} \max }$ are the water flow and its minimum and maximum limitations of the $k^{\text {th }}$ station; and $Q_{k, t}^{\text {in }}, Q_{k, t}^{\text {out }}, Q_{k, t}^{\mathrm{Gen}}, Q_{k, t}^{\mathrm{Cur}}$ are the input flow, output flow, generation flow, and curtailed water flow, respectively. $\delta^{L}, \delta^{U}$ are the minimum and maximum power variation limitations in a unit interval.

3.2. Pumped Storage System Operating Constraints. Constraints of PSH include water storage changing constraints (59), generation and pumping power constraints (60), power balance constraint (61), ramp rate constraints (62), and operating status mutually exclusive constraint (63):

$$
\left\{\begin{array}{l}
V_{t+1}^{U}=V_{t}^{U}-\Delta t \frac{\mathrm{EP}_{t}^{\mathrm{PSG}}}{\eta_{G}} \\
V_{t+1}^{L}=V_{t}^{L}+\Delta t \frac{\mathrm{EP}_{t}^{\mathrm{PSG}}}{\eta_{G}} \\
V_{t+1}^{U}=V_{t}^{U}+\Delta t \eta_{P} \mathrm{EP}_{t}^{\mathrm{PSP}} \\
V_{t+1}^{L}=V_{t}^{L}-\Delta t \eta_{P} \mathrm{EP}_{t}^{\mathrm{PSP}} \\
V^{U \min } \leq V_{t+1}^{U}, V_{t}^{U} \leq V^{U \max } \\
V^{L \min } \leq V_{i, t+1}^{L}, V_{i, t}^{L} \leq V^{L \max } \\
P_{t}^{\mathrm{PSG} \min } \leq \mathrm{EP}_{t}^{\mathrm{PSG}} \leq \mathrm{min}\left[P^{\mathrm{PSG} \max }, \frac{V_{t}^{U}}{\Delta t} \eta_{G}\right] \text { or } \mathrm{EP}_{t}^{\mathrm{PSG}}=0 \\
P^{\mathrm{PSP} \min } \leq \mathrm{EP}_{t}^{\mathrm{PSP}} \leq \min \left[P^{\mathrm{PSP} \max }, \frac{V_{t}^{L}}{\Delta t \eta_{P}}\right] \text { or } \mathrm{EP}_{t}^{\mathrm{PSP}}=0
\end{array}\right.
$$

$$
\begin{aligned}
& \left\{\begin{array}{l}
\delta_{G}^{L} \Delta t \leq \mathrm{EP}_{t+1}^{\mathrm{PSG}}-\mathrm{EP}_{t}^{\mathrm{PSG}} \leq \delta_{G}^{U} \Delta t, \\
\delta_{P}^{L} \Delta t \leq \mathrm{EP}_{t+1}^{\mathrm{PSP}}-\mathrm{EP}_{t}^{\mathrm{PSP}} \leq \delta_{P}^{U} \Delta t,
\end{array}\right. \\
& z_{t}^{\mathrm{PSG}} \cdot z_{t}^{\mathrm{PSP}}=0,
\end{aligned}
$$

where $V_{t}^{U}, V_{t}^{L}, V^{U \min }, V^{U \max }, V^{L \min }$, and $V^{L \max }$ are water storage of the upper and lower reservoir, and their minimum and maximum limitations, respectively; $\eta_{G}, \eta_{P}$ are generating efficiency and pumping efficiency; $P^{\mathrm{PSG} \text { min }}, P^{\mathrm{PSG} \max }$, $P^{\text {PSP min }}, P^{\text {PSP max }}$ are the minimum and maximum power limitations in generating and pumping status respectively; and $\delta_{G}^{L}, \delta_{G}^{U}, \delta_{P}^{L}, \delta_{P}^{U}$ are the minimum and maximum power variation limitations in generating and pumping status in a unit interval.

3.3. DC Power Flow Constraint. DC power flow constraint is taken into account in the dual-objective dispatching model:

$$
\left\{\begin{array}{l}
P_{\text {tline }}=B_{\text {diag }} L B^{-1}\left(\mathrm{EP}_{t}^{\mathrm{CH}}+\mathrm{EP}_{t}^{\mathrm{PV}}-\mathrm{EP}_{t}^{\mathrm{PSP}} z_{t}^{\mathrm{PSP}}+\mathrm{EP}_{t}^{\mathrm{PSG}} z_{t}^{\mathrm{PSG}}-P_{t}^{L}\right), \\
-\bar{P}_{\text {line }} \leq P_{\text {tline }} \leq \bar{P}_{\text {line }}, B_{\text {diag }}=\operatorname{diag}\left(\frac{1}{x_{1}}, \frac{1}{x_{2}}, \ldots, \frac{1}{x_{N}}\right),
\end{array}\right.
$$

where $B$ is the admittance coefficient matrix; $x_{n}$ is the reactance of the $n^{\text {th }}$ branch; $N$ is the number of branches in the system; $\bar{P}_{\text {line }}$ is the upper limitation of branch power.

Based on the proposed dual-objective model, the MOQPSO algorithm [41] is introduced to calculate the model. This algorithm is derived from QPSO (Quantumbehaved Particle Swarm Optimization) algorithm, and improvements from three aspects-external file set, dominant individual selection, and chaotic mutation search-are implemented to meet the efficient calculation needs of multiobjective optimization problems. The overall calculating flow is shown in Figure 2.

From Figure 2, several steps are included in the flow and described as follows. Firstly, encode each output combination scheme obtained by the PCPS method, and each particle represents a possible dispatching scheme. Secondly, evaluate and calculate the corresponding objective function of each particle and update the external file set. Thirdly, update the optimal position of individuals in history, and perform the mutation search for the individuals. Fourthly, determine the global optimal position and update the position of each particle. Then, check the termination condition and determine whether the flow continues or terminates. Finally, output the Pareto optimal solution set and the dispatching data.

\section{Case Study}

The modified IEEE 24 bus test system [42] is introduced to examine the effectiveness of the proposed probabilistic dispatching approach for the cascaded hydro- PV-PSH joint complementary power system. The test system consists of 11 renewable power stations located on buses $1,2,7,13,14,15$, $16,18,21,22$, and 23 , and their distribution is shown in 


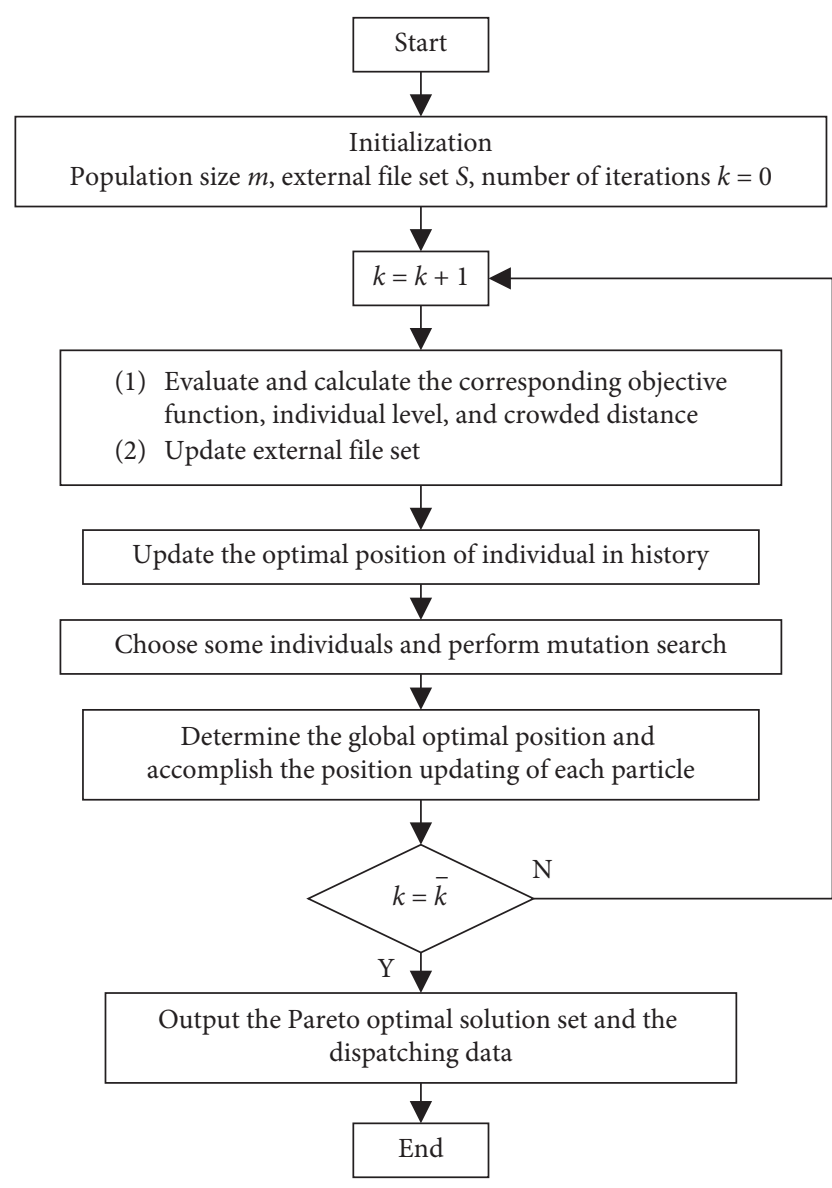

FIGURE 2: Calculating flow of MOQPSO algorithm.

Table 1. The corresponding data of each branch and loads of the test system is modified properly to accommodate the renewable generation. The details of each station are shown in Table 1. The station located at bus 7 is a runoff hydropower station, and the rest hydropower stations have the daily regulating capability. The profiles of PV output expectation in different weather conditions obtained by the PCPS approach are shown in Figure 3.

The cascade hydropower stations in the hybrid system are taken as an equivalent adjustable hydropower station, and its power output is divided into two parts: the uncertain natural runoff output and the adjustable water storage output. The former mainly depends on random runoff flow and the latter is related to the storage capacity of reservoirs. Based on historical hydraulic data of the cascade hydropower stations, the total runoff power output profiles in different seasons (wet season, normal season, and dry season) are displayed in Figure 4.

In the production simulation process, the probability distribution of the stochastic shielding factor of PV power output is considered to conform to the Beta distribution [40]. The corresponding parameters used in the production simulation process are listed in Table 2 . The power generation distribution schemes of each station and the corresponding reliability data and operating cost data can be obtained based on the proposed PCPS technique.

After obtaining the power generation distribution schemes that meet complementary requirements, the schemes are taken into the optimization process based on the MOQPSO algorithm to seek the Pareto optimal scheme. In the MOQPSO algorithm, population size $m$ is set as 500 , the number of individuals in the external file set $S$ is 30 , and the maximum number of iterations is set as 1000 . Taking the sunny day/wet season scenario as an example, Figure 5 gives its Pareto optimal front set between reliability (LOLP) and cost factor. The cost factor is calculated by the ratio of power generation cost to the total operating cost of the hybrid system (including power generation cost, penalty cost, and power purchasing cost). It can be found from the scatterplot that the comprehensive LOLP decreases gradually with the increase of cost factor, and the speed of decrease is fast in the initial stage then becomes slow gradually. When the cost factor exceeds a certain value, the LOLP will not change significantly as the cost factor increases. If the reliability is still wished to further improvement at this moment, greater economic cost will be paid, and the economic performance of the dispatching schemes will deteriorate. The generation distribution scheme at this point is believed optimal.

Four representative power generation scenarios-sunny day/wet season, rainy day/wet season, sunny day/dry season, and rainy day/dry season-are chosen, and the optimal probabilistic chronological generation dispatching schemes for cascade hydro-PV-PSH complementary power system in each scenario are presented as follows.

Analyzing the optimal dispatching schemes in the case study displayed above, some beneficial discussions are carried out as follows:

(1) The overall scale of hydropower output in the wet season has no great difference compared to that in the dry season. The influence of season variation is weakened. This is mainly attributed to the reservoir regulating capability of the cascaded hydropower system. Generally, the regulating performance of cascaded hydropower is better than that of single hydropower of the same scale [43].

(2) Because of the policy of promoting the consumption of PV resources, its energy curtailment price is set to be higher than that of hydro resources in the case study. PV power stations take the priority to generate, and the hydropower stations support and cooperate with $\mathrm{PV}$ stations to meet the loads demand.

(3) From Figures 6-9, it can be found that PSH is in pumping status in the wet season and generally occurs in the middle of the daytime. In contrast, PSH is in generating status in the dry season and occurs at night. To evaluate the economic performance of PSH in the hybrid system, four new operating scenarios without PSH are constructed and the average unit 
TABle 1: Parameters of each generator.

\begin{tabular}{|c|c|c|c|c|c|}
\hline Bus ID & Power type & Installed capacity (MW) & Reservoir capacity $\left(10^{4} \mathrm{~m}^{3}\right)$ & Water head (m) & Working flow $\left(\mathrm{m}^{3} / \mathrm{s}\right)$ \\
\hline 1 & PV-1 & 50 & - & - & - \\
\hline 2 & PV-2 & 50 & - & - & - \\
\hline 7 & $\mathrm{CH}-5$ & 20 & - & 339.09 & 7.4 \\
\hline 13 & $\mathrm{CH}-3$ & 36 & 37.2 & 91 & 33 \\
\hline 14 & - & - & - & - & - \\
\hline 15 & $\mathrm{CH}-6$ & 4.8 & 53 & 10.5 & 48.9 \\
\hline 16 & PSH & 5 & $25.6 / 23.7$ & 339.09 & 7.4 \\
\hline 18 & $\mathrm{CH}-2$ & 60 & 20.9 & 127 & 53.4 \\
\hline 21 & $\mathrm{CH}-4$ & 54 & 25.6 & 161 & 47.1 \\
\hline 22 & $\mathrm{CH}-1$ & 45 & 16.1 & 123.65 & 43.32 \\
\hline 23 & $\mathrm{CH}-7$ & 84 & 44.2 & 75 & - \\
\hline
\end{tabular}

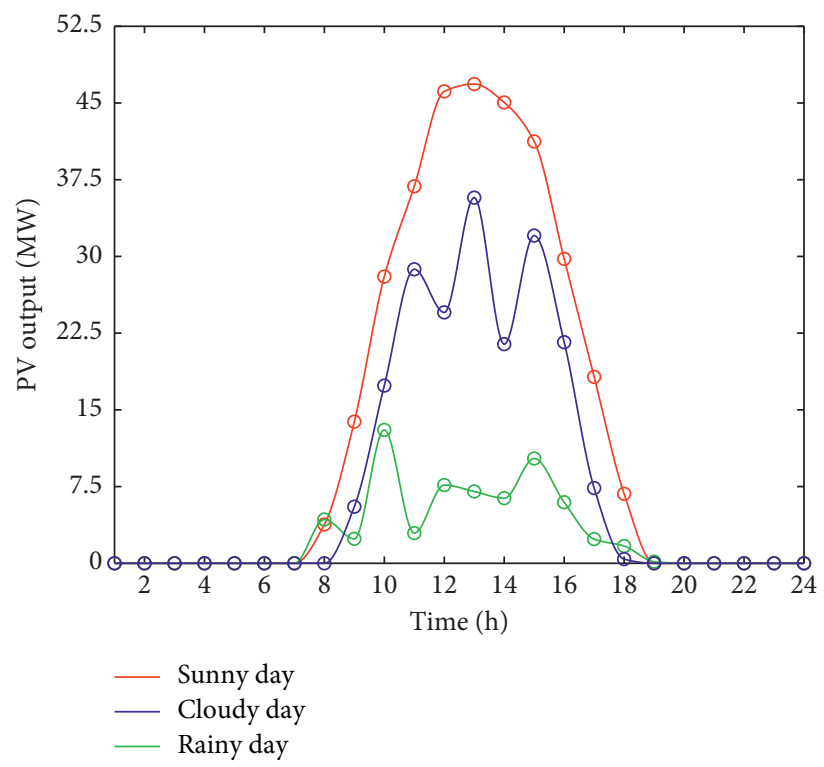

FIgUre 3: Profiles of PV output expectations.

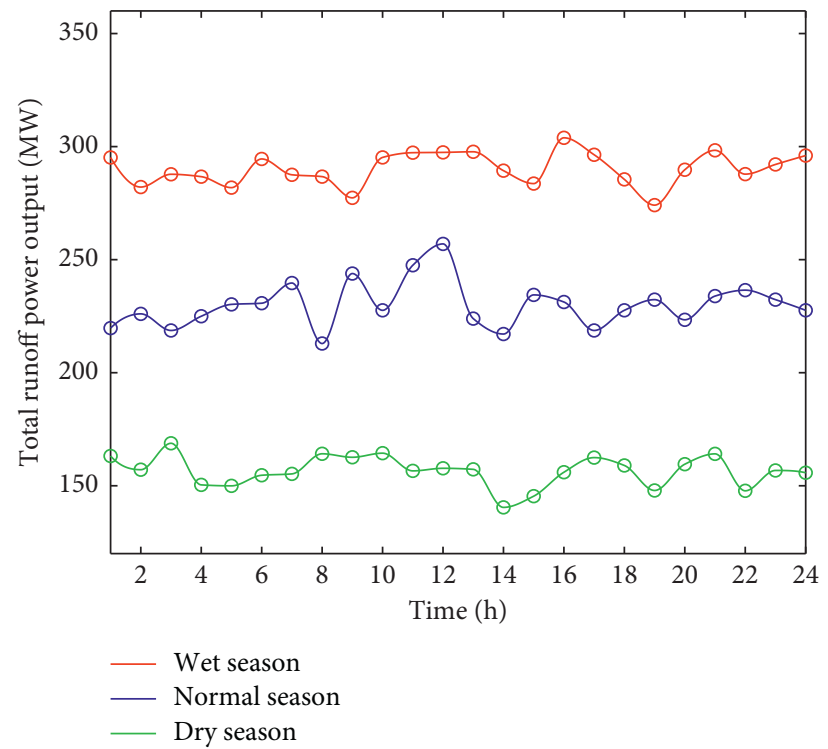

Figure 4: Total runoff power output profiles in different seasons. 
TABle 2: Parameters used in production simulation.

\begin{tabular}{lccccc}
\hline Power type & $\begin{array}{c}\text { Unit capacity } \\
(\mathrm{MW})\end{array}$ & $\begin{array}{c}\text { Unit forced outage } \\
\text { rate }\end{array}$ & $\begin{array}{c}\text { Generation price } \\
(¥ / \mathrm{MWh})\end{array}$ & $\begin{array}{c}\text { Shortage price } \\
(¥ / \mathrm{MWh})\end{array}$ & $\begin{array}{c}\text { Curtailment price } \\
(¥ / \mathrm{MWh})\end{array}$ \\
\hline $\begin{array}{l}\text { Cascade } \\
\text { hydro }\end{array}$ & 1 & 0.089 & 150 & 185 & 185 \\
\hline Power type & Longitude $\left(^{\circ}\right)$ & Latitude $\left(^{\circ}\right)$ & $\begin{array}{c}\text { Generation price } \\
(¥ / \mathrm{MWh})\end{array}$ & $\begin{array}{c}\text { Shortage price } \\
(¥ / \mathrm{MWh})\end{array}$ & $\begin{array}{c}\text { Curtailment price } \\
(¥ / \mathrm{MWh})\end{array}$ \\
\hline PV & 102.3644 & 30.99918 & 125 & 185 & 205 \\
\hline Power type & $\begin{array}{c}\text { Unit capacity } \\
(\mathrm{MW})\end{array}$ & $\begin{array}{c}\text { Unit forced outage } \\
\text { rate }\end{array}$ & $\begin{array}{c}\text { Generation price } \\
(¥ / \mathrm{MWh})\end{array}$ & $\begin{array}{c}\text { Shortage price } \\
(¥ / \mathrm{MWh})\end{array}$ & Pumping price $(¥ / \mathrm{MWh})$ \\
\hline PSH & 0.5 & 0.08 & 240 & 310 & 180 \\
\hline
\end{tabular}

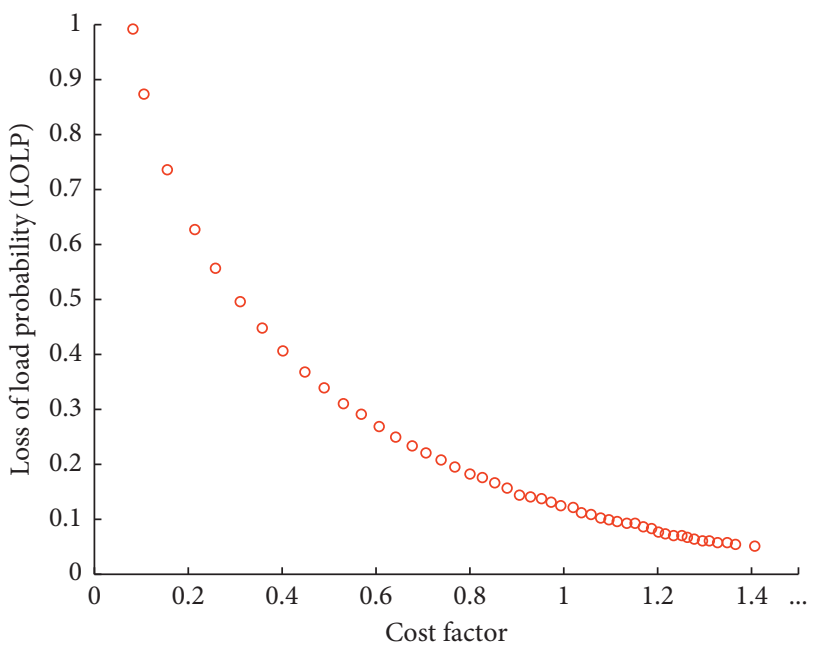

Figure 5: Pareto optimal front set of sunny day/wet season scenario.

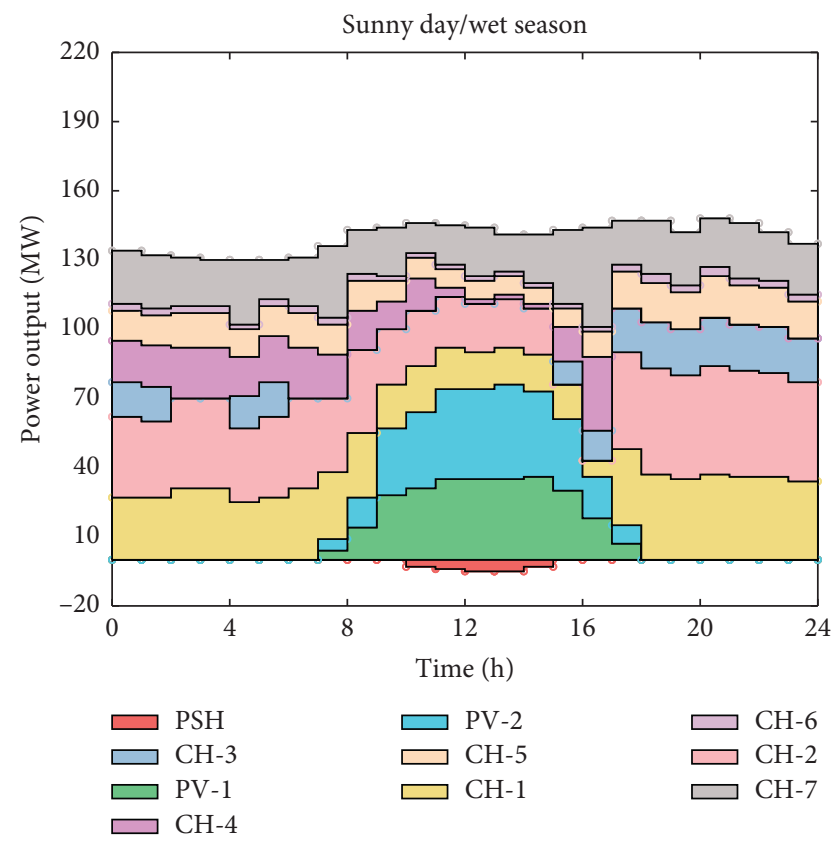

FIGURE 6: Optimized dispatching scheme in sunny day/wet season.

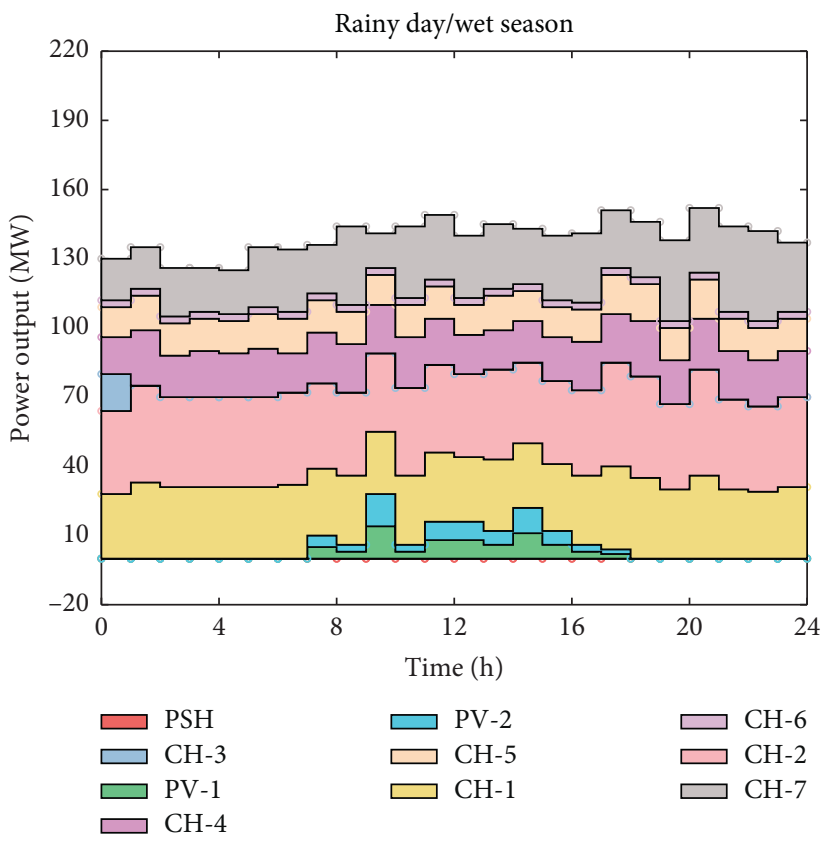

FIGURE 7: Optimized dispatching scheme in rainy day/wet season.

power generation cost of each scenario is calculated and shown in Table 3. The unit cost in the rainy day/ dry scenario is the highest, and the cost in the sunny/ wet scenario is the lowest. However, due to the application of PSH, the decline of unit cost in the rainy/dry scenario is much larger than the increases in other scenarios caused by the pumping operation. The effectiveness and value of PSH are testified from the comparison of the results.

(4) Compared with the time data that it takes about 3.7 hours to complete the same scale calculation with DP (dynamic programming, DP) technique (discrete states number is 100) in [41], it only needs less than 10 seconds to complete the optimization process using the proposed method of this paper. It has verified the feasibility and efficiency of the proposed method through comparison with the benchmark data. 


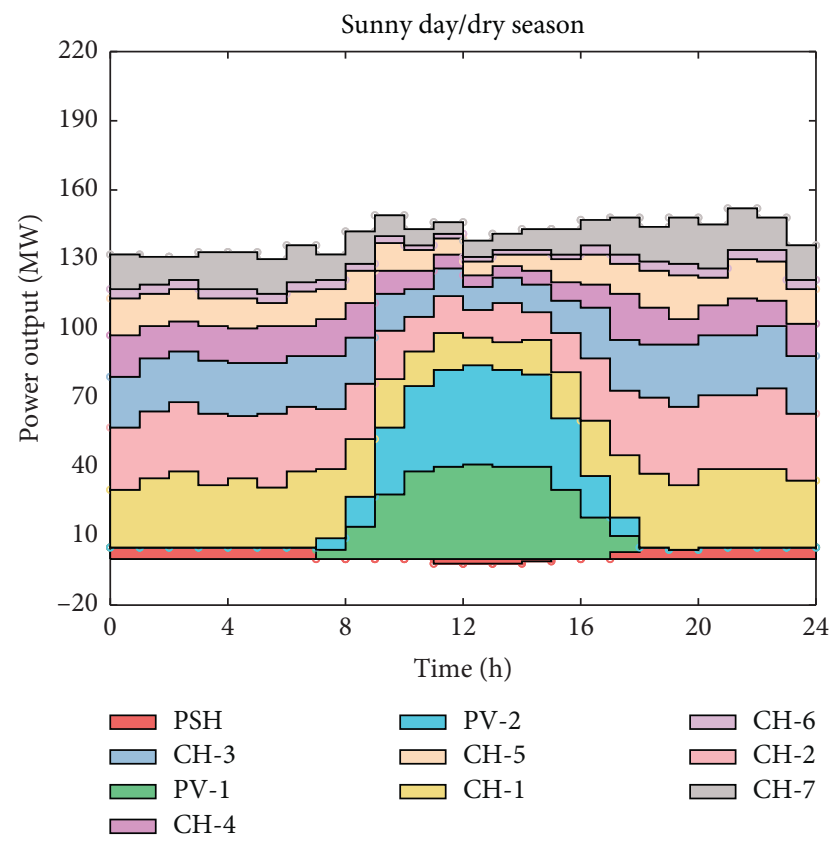

FIGURE 8: Optimized dispatching scheme in sunny day/dry season.

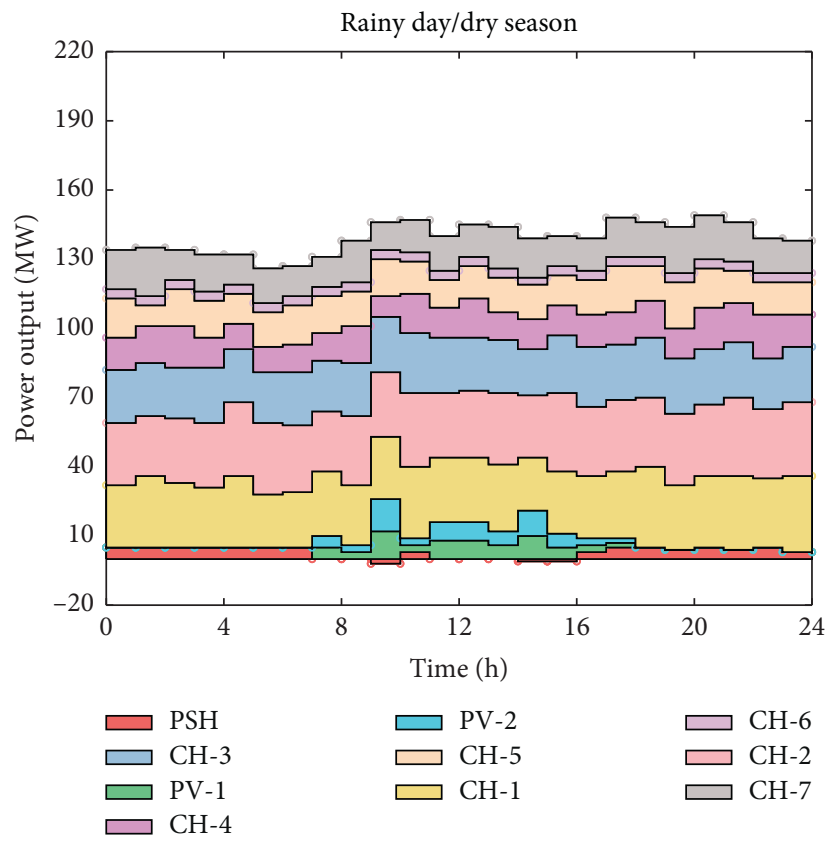

Figure 9: Optimized dispatching scheme in rainy day/dry season.

TABLE 3: Average unit power generation cost of different scenarios (¥/MWh).

\begin{tabular}{lcccc}
\hline Type & Sunny/wet & Rainy/wet & Sunny/dry & Rainy/dry \\
\hline CH-PV & 138.5945 & 147.0218 & 145.8147 & 152.3571 \\
CH-PV-PSH & 138.6383 & 147.0218 & 145.8322 & 151.9286 \\
\hline
\end{tabular}




\section{Conclusions}

Focusing on the uncertainty and reliability problems in the power generation dispatching process of multiple renewables combined complementary system, a dual-stage probabilistic coordinated dispatching method is proposed in this paper. First, a modified probabilistic chronological production simulation method is proposed to simulate the uncertain power output characteristics of multiple renewables, such as cascaded hydropower, $\mathrm{PV}$, and $\mathrm{PSH}$, and to obtain the complementary dispatching schemes for the cascaded hydro-PV-PSH system and the reliability and economic indexes of each scheme. On this basis, a dual-objective optimization model of dispatching schemes considering the reliability and economic performance of power generation is introduced. Meanwhile, the MOQPSO algorithm is applied to calculate the optimal Pareto solution set. The results of the case study have verified the effectiveness and calculating efficiency of the proposed dispatching method. The main contributions of the paper are listed as follows:

(1) A modified probabilistic chronological production simulation method for uncertain timing-output simulation of multiple renewables is proposed.

(2) An efficient dual-objective optimization method for power generation dispatching schemes of renewable energy is obtained.

(3) A dispatching method for cascaded hydropower system that the overall probabilistic chronological production simulation is performed first, then implementing the interstage distribution, is proposed to cope with the multiple uncertainties and coupling.

(4) The feasibility and complementary capabilities of hydroPV combined power generation have been further verified. The function of PSH to regulate the uncertain complementary output of renewables has been testified.

This paper mainly concerns the multiple uncertainties and coupling of multiple renewables combined power system and sheds less light on the benefits of each generation member. Therefore, how to maximize the benefits of each member to fully motivate them to participate in the complementary power generation and thus generate an optimized dispatching scheme for the cascaded hydro-PV-PSH complementary system will be the next research focus.

\section{Data Availability}

The technical route, the model structure, and the parameters of case study are available from the corresponding author upon request.

\section{Conflicts of Interest}

The authors have no conflicts of interest.

\section{Acknowledgments}

This work was supported by the National Key R\&D Program of China (2018YFB0905200)-Research and application demonstration on complementary combined power generation technology for distributed PV and cascade hydropower.

\section{References}

[1] T. Niknam and B. B. Firouzi, "A practical algorithm for distribution state estimation including renewable energy sources," Renewable Energy, vol. 34, no. 11, pp. 2309-2316, 2009.

[2] R. Hosseinalizadeh, H. Shakouri G, M. S. Amalnick, and P. Taghipour, "Economic sizing of a hybrid (PV-WT-FC) renewable energy system (HRES) for stand-alone usages by an optimization-simulation model: case study of Iran," Renewable and Sustainable Energy Reviews, vol. 54, pp. 139-150, 2016.

[3] D. B. Nelson, M. H. Nehrir, and C. Wang, "Unit sizing and cost analysis of stand-alone hybrid wind/PV/fuel cell power generation systems," Renewable Energy, vol. 31, no. 10, pp. 1641-1656, 2006.

[4] R. Siddaiah and R. P. Saini, "A review on planning, configurations, modeling and optimization techniques of hybrid renewable energy systems for off grid applications," Renewable and Sustainable Energy Reviews, vol. 58, pp. 376-396, 2016.

[5] R. Siddaiah, "Resources, configurations, and soft computing techniques for power management and control of PV/wind hybrid system," Renewable and Sustainable Energy Reviews, vol. 69, pp. 129-143, 2017.

[6] S. Upadhyay and M. P. Sharma, "A review on configurations, control and sizing methodologies of hybrid energy systems," Renewable and Sustainable Energy Reviews, vol. 38, pp. 47-63, 2014.

[7] Z. Abdmouleh, A. Gastli, L. Ben-Brahim, M. Haouari, and N. A. Al-Emadi, "Review of optimization techniques applied for the integration of distributed generation from renewable energy sources," Renewable Energy, vol. 113, pp. 266-280, 2017.

[8] W. D. Kellogg, M. H. Nehrir, G. Venkataramanan, and V. Gerez, "Generation unit sizing and cost analysis for standalone wind, photovoltaic, and hybrid wind/PV systems," IEEE Transactions on Energy Conversion, vol. 13, no. 1, pp. 70-75, 1998.

[9] J. Samaniego, F. Alija, S. Sanz, C. Valmaseda, and F. Frechoso, "Economic and technical analysis of a hybrid wind fuel cell energy system," Renewable Energy, vol. 33, no. 5, pp. 839-845, 2008.

[10] P. Zhou, R. Y. Jin, and L. W. Fan, "Reliability and economic evaluation of power system with renewables: a review," $R e$ newable and Sustainable Energy Reviews, vol. 58, pp. 537-547, 2016.

[11] S. Goel and R. Sharma, "Performance evaluation of stand alone, grid connected and hybrid renewable energy systems for rural application: a comparative review," Renewable and Sustainable Energy Reviews, vol. 78, pp. 1378-1389, 2017.

[12] T. Tezer, R. Yaman, and G. Yaman, "Evaluation of approaches used for optimization of stand-alone hybrid renewable energy systems," Renewable and Sustainable Energy Reviews, vol. 73, pp. 840-853, 2017.

[13] A. A. Eras-Almeida and M. A. Egido-Aguilera, "Hybrid renewable mini-grids on non-interconnected small islands: review of case studies," Renewable and Sustainable Energy Reviews, vol. 116, 2019.

[14] M. Wu, L. Kou, X. Hou, Y. Ji, B. Xu, and H. Gao, “A bi-level robust planning model for active distribution networks 
considering uncertainties of renewable energies," International Journal of Electrical Power \& Energy Systems, vol. 105, pp. 814-822, 2019.

[15] A. Zakaria, F. B. Ismail, M. S. H. Lipu, and M. A. Hannan, "Uncertainty models for stochastic optimization in renewable energy applications," Renewable Energy, vol. 145, pp. 15431571, 2020.

[16] C. Voyant, G. Notton, S. Kalogirou et al., "Machine learning methods for solar radiation forecasting: a review," Renewable Energy, vol. 105, pp. 569-582, 2017.

[17] Z. Jun, L. Junfeng, W. Jie, and H. W. Ngan, “A multi-agent solution to energy management in hybrid renewable energy generation system," Renewable Energy, vol. 36, no. 5, pp. 1352-1363, 2011.

[18] M. Nazari-Heris, B. Mohammadi-Ivatloo, and G. B. Gharehpetian, "Short-term scheduling of hydro-based power plants considering application of heuristic algorithms: a comprehensive review," Renewable and Sustainable Energy Reviews, vol. 74, pp. 116-129, 2017.

[19] K. Nghitevelekwa and R. C. Bansal, "A review of generation dispatch with large-scale photovoltaic systems," Renewable and Sustainable Energy Reviews, vol. 81, pp. 615-624, 2018.

[20] K. Sopian, A. Zaharim, Y. Ali, Z. M. Nopiah, J. A. Razak, and N. S. Muhammad, "Optimal operational strategy for hybrid renewable energy system using genetic algorithms," WSEAS Transactions on Mathematics, vol. 7, no. 4, pp. 130-140, 2008.

[21] M. S. Javed, T. Ma, J. Jurasz, and M. Y. Amin, "Solar and wind power generation systems with pumped hydro storage: review and future perspectives," Renewable Energy, vol. 148, pp. 176-192, 2020.

[22] T. Mahto and V. Mukherjee, "Energy storage systems for mitigating the variability of isolated hybrid power system," Renewable and Sustainable Energy Reviews, vol. 51, pp. 1564-1577, 2015.

[23] J. Hu, R. Harmsen, W. Crijns-Graus, and E. Worrell, "Barriers to investment in utility-scale variable renewable electricity (VRE) generation projects," Renewable Energy, vol. 121, pp. 730-744, 2018.

[24] M. K. C. Marwali, M. Haili, S. M. Shahidehpour, and K. H. Abdul-Rahman, "Short term generation scheduling in photovoltaic-utility grid with battery storage," IEEE Transactions on Power Systems, vol. 13, no. 3, pp. 1057-1062, 1998.

[25] X. S. Zhang, Z. Xu, T. Yu, B. Yang, and H. Z. Wang, "Optimal mileage based AGC dispatch of a GenCo," Ieee Transactions on Power Systems, vol. 35, no. 4, pp. 25162526, 2020.

[26] X. S. Zhang, T. Tan, B. Zhou, T. Yu, B. Yang, and X. M. Huang, "Adaptive distributed auction-based algorithm for optimal mileage based AGC dispatch with high participation of renewable energy," International Journal of Electrical Power \& Energy Systems, vol. 124, 2021.

[27] M. Grami, M. Rekik, and L. Krichen, "Power dispatch strategy for interconnected microgrids based hybrid renewable energy system," International Journal of Renewable Energy Research, vol. 8, no. 2, pp. 838-850, 2018.

[28] S. S. Reddy and P. R. Bijwe, "Day-ahead and real time optimal power flow considering renewable energy resources," International Journal of Electrical Power \& Energy Systems, vol. 82, pp. 400-408, 2016.

[29] A. Hassan, R. Mieth, M. Chertkov, D. Deka, and Y. Dvorkin, "Optimal load ensemble control in chance-constrained optimal power flow," IEEE Transactions on Smart Grid, vol. 10, no. 5, pp. 5186-5195, 2019.
[30] A. Lorca and X. A. Sun, "Adaptive robust optimization with dynamic uncertainty sets for multi-period economic dispatch under significant wind," IEEE Transactions on Power Systems, vol. 30, no. 4, pp. 1702-1713, 2015.

[31] Z. Hu, Z. Yan, and J. Wen, "Planning and dispatching of hybrid renewable power system based on portfolio theory," 2011.

[32] S. Eftekharnejad, G. T. Heydt, and V. Vittal, "Optimal generation dispatch with high penetration of photovoltaic generation," IEEE Transactions on Sustainable Energy, vol. 6, no. 3, pp. 1013-1020, 2015.

[33] R. Booth, "Power system simulation model based on probability analysis," IEEE Transactions on Power Apparatus and Systems, vol. 91, no. 1, pp. 62-69, 1972.

[34] M. Lin, A. Breipohl, and F. Lee, "Comparison of probabilistic production cost simulation methods," IEEE Transactions on Power Systems, vol. 4, no. 4, pp. 1326-1334, 1989.

[35] X. Wang, "Equivalent energy function approach to power system probabilistic modeling," IEEE Transactions on Power Systems, vol. 3, no. 3, pp. 823-829, 1988.

[36] Q. Xia, S. Wang, and N. Xiang, "The probabilistic power system production simulation based on chronological load curve," Proceedings of the CSEE, vol. 14, no. 3, pp. 21-28, 1994.

[37] M. Alata, M. A. Al-Nimr, and Y. Qaroush, "Developing a multipurpose sun tracking system using fuzzy control," Energy Conversion and Management, vol. 46, no. 7-8, pp. 1229-1245, 2005.

[38] F. S. Higgs, Principles of Solar Engineering: By Frank Kreith and Jan F. Kreider, McGraw-Hill Book Company, New York, NY, USA, 1978.

[39] B. Y. H. Liu and R. C. Jordan, "The interrelationship and characteristic distribution of direct, diffuse and total solar radiation," Solar Energy, vol. 4, no. 3, pp. 1-19, 1960.

[40] K. C. Zhang Xi, "Analysis of mid/long term random characteristic of photovoltaic power generation," Automation of Electric Power Systems (In Chinese), vol. 38, pp. 6-12, 2014.

[41] Z.-k. Feng, W.-j. Niu, and C.-t. Cheng, "Multi-objective quantum-behaved particle swarm optimization for economic environmental hydrothermal energy system scheduling," Energy, vol. 131, pp. 165-178, 2017.

[42] C. Grigg, P. Wong, P. Albrecht et al., "Reliability test system task, "the IEEE reliability test system-1996," IEEE Transactions on Power Systems, vol. 14, no. 3, pp. 1010-1018, 1999.

[43] C. Cheng, F. Chen, G. Li et al., "Reform and renewables in China: the architecture of Yunnan's hydropower dominated electricity market," Renewable and Sustainable Energy Reviews, vol. 94, pp. 682-693, 2018. 